12th International Symposium on Cosmology and

Particle Astrophysics (CosPA 2015)

International Journal of Modern Physics: Conference Series

Vol. 43 (2016) 1660200 (8 pages)

(C) The Author(s)

DOI: $10.1142 / \mathrm{S} 2010194516602003$

\title{
Electroweak Baryogenesis with Anomalous Higgs Couplings
}

\author{
Archil Kobakhidze*, Lei $\mathrm{Wu}^{\dagger}$ and Jason Yue \\ ARC Centre of Excellence for Particle Physics at the Terascale, \\ School of Physics, The University of Sydney, NSW 2006, Australia \\ *archil.kobakhidze@sydney.edu.au \\ †lei.wu1@sydney.edu.au \\ ¥jason.yue@sydney.edu.au
}

Published 7 July 2016

\begin{abstract}
In non-linear realisation of the electroweak gauge symmetry, the LHC Higgs boson can be assumed to be a singlet under $S U(2)_{L} \otimes U(1)_{Y}$. In such scenario, the Standard Model particle content can be kept but new sets of couplings are allowed. We identify a range of anomalous Higgs cubic and the $\mathcal{C P}$-violating Higgs-top quark couplings that leads to first order phase transition and successful baryogenesis at the electrweak scale.
\end{abstract}

Keywords: Electroweak baryogensis; Higgs self coupling; $\mathcal{C P}$-violating Yukawa couplings.

\section{Introduction}

The $125 \mathrm{GeV}$ Higgs boson are showing properties consistent ${ }^{1}$ with the Standard Model Higgs at the Large Hadron Collider (LHC). This has important implications on explaining the observed matter-antimatter asymmetry in the universe through baryogenesis during the electroweak phase transition. ${ }^{2}$ Such asymmetry is measured $^{3,4}$ in terms of the parameter:

$$
\eta_{B}:=n_{B} / s \approx 8.6 \times 10^{-11},
$$

where net baryon number $n_{B}$ is normalised by the entropy density $s$. It has been established a Higgs mass $\gtrsim 70 \mathrm{GeV}$ does not result in a first-order phase transition and that the standard Cabibbo-Kobayashi-Maskawa $\mathcal{C P}$-violation is insufficient to explain the current baryon asymmetry. This asymmetry therefore suggest the existence of new physics beyond the Standard Model.

We investigate feasibility of efficient baryogenesis at the electroweak scale within the non-linear realisation of the electroweak gauge symmetry. Such framework describes the physical Higgs boson as a singlet under $S U(2)_{L} \otimes U(1)_{Y}$ and hence

This is an Open Access article published by World Scientific Publishing Company. It is distributed under the terms of the Creative Commons Attribution 4.0 (CC-BY) License. Further distribution of this work is permitted, provided the original work is properly cited. 
admits anomalous tree-level cubic Higgs interactions and $\mathcal{C} \mathcal{P}$-violating Higgs-Top interactions amongst others. The couplings are not tightly constraint by current data and so play a significant role in the electroweak phase transition and baryogenesis. In what follows, we discuss such model in detail.

\section{Description of the model}

In non-linear realisation ${ }^{5}$ of the electroweak symmetry, the Higgs field $h$ is not obliged to form the electroweak doublet irreducible representation as in the SM. Instead, the physical Higgs boson would be identified as the physical excitation of the singlet $\rho(x)=v+h(x)$ and the three would-be Goldstone bosons $\pi_{i}(x)$ which parameterise $S U(2) \otimes U(1)_{Y} / U(1)_{Q}$ coset space form a nonlinear field $\mathcal{X}(x)$ :

$$
\mathcal{X}(x)=e^{\frac{i}{2} \pi_{i}(x) T_{i}}\left(\begin{array}{l}
0 \\
1
\end{array}\right)
$$

where $T_{i}=\delta_{i 3} \mathbb{1}+\sigma_{i}$ are the three broken generators with $\sigma_{i}$ being Pauli matrices. The SM Higgs doublet is recovered as the composite field ${ }^{\mathrm{a}}$ :

$$
H(x)=\frac{\rho(x)}{\sqrt{2}} \mathcal{X}(x) .
$$

New interactions beyond those present in SM are possible in the non-linear paramaterisation of eletroweak symmetry breaking. Here the relevant interactions to electroweak baryogenesis are $(1)$ the $\mathcal{C P}$-violating Higgs-top Yukawa interactions:

$$
\mathcal{L}_{\text {Higgs-Top }}=-\left[m_{t}^{\prime}+y_{t} \mathrm{e}^{i \xi} \rho / \sqrt{2}\right] \bar{Q}_{L} \tilde{\mathcal{X}} t_{\mathrm{R}}+\text { h.c. },
$$

where $Q_{L}=\left(t_{L}, b_{L}\right)^{\mathrm{T}}$ is the third generation left-handed quark doublet, $\tilde{\mathcal{X}}=i \sigma^{2} \mathcal{X}^{*}$, $m_{t}^{\prime}$ is an additional mass parameter and is a complex coupling, and (2) a tree-level cubic term in the Higgs potential

$$
V^{(0)}(\rho)=-\frac{\mu^{2}}{2} \rho^{2}+\frac{\kappa}{3} \rho^{3}+\frac{\lambda}{4} \rho^{4},
$$

The constraints on the top-Higgs sector still allows ${ }^{6} \mathcal{C} \mathcal{P}$-phases $\xi \in[-0.6 \pi, 0.6 \pi]$ and modulus $y_{t} \in\left[0.4 y_{t}^{S M}, 1.6 y_{t}^{S M}\right]$, where $y_{t}^{S M}=\sqrt{2} m_{t} / v$. The contribution of such sector to the electron electric dipole moments is consistent ${ }^{7}$ with the experimental constraints on the electron EDM. ${ }^{8}$ The Higgs cubic couplings which is affected by $\kappa$ is currently relatively unconstrained. These interactions respectively gives additional $\mathcal{C P}$-violation to the top-Higgs sector and enhancing the first-order electroweak phase transition, subsequently can lead to successful electroweak baryogenesis.

${ }^{a}$ We note that if $\rho(x)$ field is to be identified with the modulus of the electroweak doublet field, $\rho^{2}=H^{\dagger} H$, it should be restricted to positive $(\rho>0)$ or negative $(\rho<0)$ values only. 
We assume that the scalar potential has a global minimum for a non-zero vacuum expectation value of the Higgs field $\rho$ (see the next section):

$$
\langle\rho\rangle=v,|v| \approx 246 \mathrm{GeV},
$$

so that describes the squared tree level Higgs mass $m_{h}^{2}=(125 \mathrm{GeV})^{2}$ is defined as the second derivative of $(5)$ at $\rho=v$.

The absolute value of the vacuum expectation value in (6) is fixed to the standard value since the Higgs interactions with the electroweak gauge bosons are assumed to be the same as in SM, i.e.,

$$
\frac{\rho^{2}}{2}\left(D_{\mu} \mathcal{X}\right)^{\dagger} D^{\mu} \mathcal{X}
$$

where $D_{\mu}$ is an $S U(2) \times U(1)_{Y}$ covariant derivative. The auxiliary top parameter $m_{t}^{\prime}$ is defined by $\xi$ and $y_{t}$, and the physical top quark mass $m_{t} \approx 173 \mathrm{GeV}$ :

$$
m_{t}^{\prime( \pm)}=\frac{1}{2}\left( \pm \sqrt{4 m_{t}^{2}-2 y_{t}^{2} v^{2} \sin ^{2} \xi}-\sqrt{2} y_{t} v \cos \xi\right) .
$$

The electroweak precision observables parametrized through the $S, T, U$ oblique parameters are essentially unaffected in our mode as they depend on particle masses and we have not introduced any new particle.

\section{The electroweak phase transition}

The presence of the cubic term in the tree-level Higgs potential (5) and the anomalous Higgs-top Yukawa couplings (4) significantly alter the Higgs vacuum configuration. The 1-loop finite temperature Higgs effective potential where only leading $T^{2}$-dependence on temperature is kept:

$$
V_{T}(\rho, T)=V^{(0)}(\rho)+\left(3 g_{2}^{2}+g_{1}^{2}+4 \lambda+4 y_{t}^{2}\right) \frac{T^{2} \rho^{2}}{2}+\left(\kappa+3 \sqrt{2} y_{t} m_{t}^{\prime} \cos \xi\right) \frac{T^{2} \rho}{12},
$$

where $g_{2,1}$ are $S U(2)$ and $U(1)_{Y}$ electroweak gauge couplings. Here, $V^{(0)}(\rho)$ is the tree level potential at zero temperature given in (5) which may be used to rewrite the mass parameter $\mu^{2}$ and the quartic coupling $\lambda$ in terms of the (tree level) Higgs mass, $m_{h} \approx 125 \mathrm{GeV}$, the Higgs vacuum expectation value $v(6)$ and the cubic coupling $\kappa$ :

$$
\begin{aligned}
\mu^{2} & =\frac{1}{2}\left(m_{h}^{2}+v \kappa\right), \\
\lambda & =\frac{1}{2 v^{2}}\left(m_{h}^{2}-v \kappa\right) .
\end{aligned}
$$

The potentially must be bounded from below, that is $\lambda>0$ and, hence, $v \kappa<m_{h}^{2}$. There are three cases to consider: (i) when the mass parameter is non-tachyonic $\left(\mu^{2}<0\right)$, the electroweak symmetry breaking minimum $(6)$ is realised as an absolute minimum of the potential if $-3 m_{h}^{2}<v \kappa<-m_{h}^{2}$, with $\rho=0$ being a local minima. (ii) when the mass parameter is tachyonic, the minimum in (6) is realised if $-m_{h}^{2}<$ $v \kappa<0$, with the trivial configuration assuming local maximum and (iii) For $\mu^{2}=$ 
$0\left(v=-\frac{\kappa}{\lambda}=-m_{h}^{2} / \kappa\right)$, where the trivial configuration is an inflection point of the potential.

One expects that the above tree-level analysis to be modified insignificantly in perturbative analysis, except when the tree level cubic parameter is small, $\kappa \approx 0$. In this case the radiative corrections induced by the anomalous Higgs-top Yukawa interactions (4) must be taken into account by the substitution:

$$
\kappa \rightarrow \kappa-\frac{3 \sqrt{2} m_{t}^{\prime} y_{t}^{3} \cos \xi}{16 \pi^{2}}\left[2 \cos ^{2} \xi+3 \ln \left(\frac{m_{t}^{\prime 2}}{v^{2}}\right)\right] .
$$

In (9), a term linear in field $\rho$ appears in additional to the the standard $\sim T^{2} \rho^{2}$ thermal correction to the thermal Higgs potential. This means that the gauge symmetry is never restored in our framework, instead the expectation value saturates at non-zero value for large temperatures $T \gg \rho$. This is illustrated in Fig. 1 where the thermal effective potential is plotted for various temperatures.

We have analysed the full one-loop thermal effective potential and found a parameter area where the above requirements for the phase transition are satisfied. The critical temperature $T_{c}$ and thermal vacuum expectation values, $v_{T_{c}}^{s}$ and $v_{T_{c}}^{b r}$ for various anomalous couplings $\kappa, y_{t}$ are given for $\xi=0.25 \pi$ in Table 1 . Such $\xi$ value remains consistent with the bounds obtained in our previous work. ${ }^{6}$

\subsection{The electroweak sphaleron rate}

The above observation has an important implication for the baryogenesis scenario. On the one hand, we require strongly first-order phase transition with sphaleron effects $^{9,13}$ to be suppressed in the broken phase, i.e. $v_{T_{c}}^{b r} / T_{c}>1$. On the other hand, sphalerons must be effective in the 'symmetric' phase, i.e. $v_{T_{c}}^{s} / T_{c}<1$. Here $T_{c}$

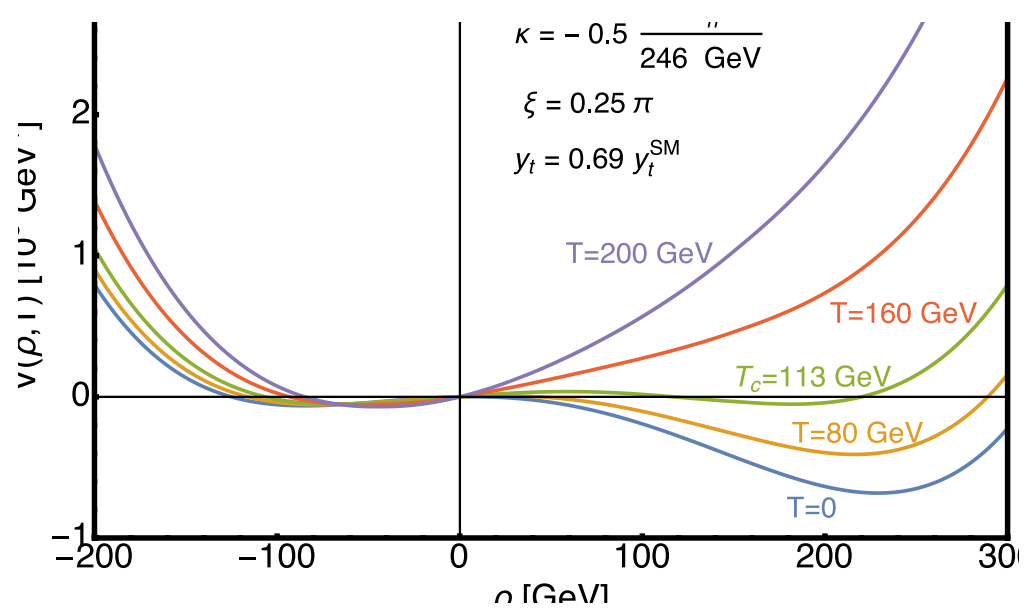

Fig. 1. Thermal effective potential at various temperatures. Non-zero thermal ground state $v_{T}^{s}$ persists at high temperatures. 
Table 1. The thermal expectation values $v_{T_{c}}^{s}, v_{T_{c}}^{b r}$ and the critical temperature $T_{c}$ for various values of $\kappa, y_{t}$ and $\xi$ corresponding to the strong first-order phase transition. For smaller $\kappa$ value the transition is a crossover or of the second-order and for larger values, the thermal and quantum correction cannot recover the electroweak symmetry breaking minimum (6). We use $m_{t}^{\prime(+)}$ from Eq. (8), as no first-order phase transition is found for $m_{t}^{\prime(-)}$ and positive $v>0$.

\begin{tabular}{|c|c|c|c|c|}
\hline \multirow{2}{*}{$\kappa|v| / m_{h}^{2}$} & & \multicolumn{3}{|c|}{$|\xi|=0.25 \pi$} \\
\hline & & $y_{t}=0.62 y_{t}^{S M}$ & $y_{t}=0.69 y_{t}^{S M}$ & $y_{t}=0.76 y_{t}^{S M}$ \\
\hline-0.1 & $\begin{array}{c}v_{T_{c}}^{s}, v_{T_{c}}^{b r} \\
T_{c}\end{array}$ & $\begin{array}{c}-197 ., 224 \\
72.9\end{array}$ & $\begin{array}{c}-194 ., 219 . \\
75.7\end{array}$ & $\begin{array}{c}-187 ., 213 . \\
81.2\end{array}$ \\
\hline-0.5 & $\begin{array}{c}v_{T_{c}}^{s}, v_{T_{c}}^{b r} \\
T_{c}\end{array}$ & $\begin{array}{c}-73.4,188 \\
114 .\end{array}$ & $\begin{array}{c}-67.5,182 . \\
113 .\end{array}$ & $\begin{array}{c}-58.8,174 \\
113 .\end{array}$ \\
\hline-1.0 & $\begin{array}{c}v_{T_{c}}^{s}, v_{T_{c}}^{b r} \\
T_{c} \\
\end{array}$ & $\begin{array}{c}-26.6,196 . \\
104 .\end{array}$ & $\begin{array}{c}-23.3,192 . \\
102 .\end{array}$ & $\begin{array}{c}-18.7,187 . \\
101 .\end{array}$ \\
\hline-1.5 & $\begin{array}{c}v_{T_{c}}^{s}, v_{T_{c}}^{b r} \\
T_{c}\end{array}$ & $\begin{array}{c}-13.0,212 . \\
86.3\end{array}$ & $\begin{array}{c}-11.2,209 . \\
83.9\end{array}$ & $\begin{array}{c}-9.03,206 . \\
81.9\end{array}$ \\
\hline-2.0 & $\begin{array}{c}v_{T_{c}}^{s}, v_{T_{c}}^{b r} \\
T_{c}\end{array}$ & $\begin{array}{c}-7.79,226 . \\
58.7\end{array}$ & $\begin{array}{c}-6.72,224 . \\
56.0\end{array}$ & $\begin{array}{c}-5.59,221 \\
54.2\end{array}$ \\
\hline
\end{tabular}

denotes a critical temperature of the phase transition defined as: $v_{T_{c}}^{s}=v_{T_{c}}^{b r}$. The finite temperature electroweak sphaleron rate within a thermal volume $V=1 / T^{3}$ in the case of non-zero Higss thermal vacuum expectation value $v_{T}$ is given by: ${ }^{10,11}$

$$
\Gamma_{s p h} \approx \frac{4 x \omega_{-}}{g_{2}\left|v_{T}\right| T^{3}}\left(\frac{\alpha_{w} T}{4 \pi}\right)^{4} \mathcal{N}_{t r}(\mathcal{N} \mathcal{V})_{r o t}\left(\frac{4 \pi\left|v_{T}\right|}{g_{2} T}\right)^{7} \exp \left(-\frac{E_{s p h}(T)}{T}\right)
$$

Here $\alpha_{w}=g_{2}^{2} / 4 \pi \approx 1 / 29.5$ is the weak isospin fine structure constant; $\omega_{-} \approx$ $g_{2}|v|$ is a dynamical pre-factor which is related to the eigenvalue of the sphaleron fluctuation operator. The factors $\mathcal{N}_{t r} \approx 26$ and $(\mathcal{N} \mathcal{V})_{\text {rot }} \approx 5.3 \times 10^{3}$ are related to the normalisation of the translational and rotational zero-modes and $x \approx 0.03$ contains the contributions of the positive modes of the fluctuation operator ${ }^{10,11, b}$. Finally, the finite temperature sphaleron energy is given by:

$$
E_{s p h}(T)=\frac{4 \pi\left|v_{T}\right|}{g_{2}} B\left(g_{2}, \lambda, \kappa\right) \sim 37\left|v_{T}\right|
$$

where $B\left(g_{2}, \lambda, \kappa\right) \in[1.97,1.85]$ when $\kappa \in\left[-2.5 m_{h}^{2} /|v|, 0\right]$ is a weakly varying function staying close to the SM value configuration $B\left(g_{2}, \lambda, 0\right) \approx 1.97$, with $\lambda$ fixed as in Eq. (11). The second approximation of (14) is applicable for $-\kappa \in[6,160] \mathrm{GeV}$.

${ }^{\mathrm{b}}$ Although the numerical values for the above factors are estimated within the SM for $\sqrt{\lambda}=g_{2}$, it should apply to our case when the sphaleron solution in our case does not differ much from the one in the SM, provided that the $|\kappa|$ is not too large. According to (11), the Higgs quartic coupling $\lambda$ in our case is larger than the Standard Model one $\lambda^{S M} \approx 0.129$, hence $\sqrt{\lambda} / g_{2} \sim 0.7-1$ for $|\kappa| \sim 30-160 \mathrm{GeV}$ 
In the symmetric phase the sphaleron rate will avoid suppression during the phase transition by demanding (13) is comparably larger than the Standard Model rate $\Gamma^{S M} \sim \alpha_{w}^{4} T$ at a critical temperature $T=T_{c}$, we obtain:

$$
0.055\left(\frac{\left|v_{T_{c}}^{s}\right|}{|v|}\right)^{1 / 7} \lesssim \frac{\left|v_{T_{s}}^{s}\right|}{T_{c}} \lesssim 1 .
$$

where the left bound avoids exponential suppression and the right bound is when the non-exponential term dominates at small $v_{T_{s}}^{s} / T_{c}$ and the exponential factor is $\mathcal{O}(1)$. The sphalerons become ineffective inside the bubble when $\left|v_{T_{c}}^{b r}\right| / T_{c} \gtrsim 1$.

\section{Computing the baryon asymmetry}

A qualitative picture of the electroweak baryogenesis may be addressed in the charge transport mechanism. ${ }^{12}$ The non-equilibrium electroweak phase transition proceeds through the nucleation and subsequent expansion of bubbles of the broken phase within the surrounding plasma in the symmetric phase. Plasma particles scattering off and diffuses from the bubble wall to generate a chiral excess $n_{L}$ in front of the wall, through $\mathcal{C P}$-violating interactions. The associated $\mathcal{C P}$ asymmetries is capture by the Higgs phase and processed by the electroweak sphaleron ${ }^{9,13}$ into a net baryon asymmetry. The sphaleron transitions must be sufficiently suppressed inside the bubble to avoid the wash-out of the generated baryon asymmetry.

We solve the set of coupled transport equations ${ }^{14}$ which gives quantitative description of the evolution of the various net particle number densities the above scenario. The final net baryon density is then: ${ }^{7}$

$$
\begin{aligned}
n_{B}= & \left(-\frac{3 \Gamma_{w s}}{v_{w}}\right)\left(-\frac{3 \bar{D}^{-1}}{64 \Gamma_{s s}}\right)\left[D_{q} \int_{-\infty}^{0} d z S_{t}^{C P V}(z)\right. \\
& +\frac{D_{q} k_{+}^{2}-v_{w} k_{+}}{k_{+}-k_{-}} \int_{-\infty}^{0} d z \int_{-\infty}^{z} d z^{\prime} e^{k_{+}\left(z-z^{\prime}\right)} S_{t}^{C P V}\left(z^{\prime}\right) \\
& \left.+\frac{D_{q} k_{-}^{2}-v_{w} k_{-}}{k_{+}-k_{-}} \int_{-\infty}^{0} d z \int_{z}^{\infty} d z^{\prime} e^{k_{-}\left(z-z^{\prime}\right)} S_{t}^{C P V}\left(z^{\prime}\right)\right],
\end{aligned}
$$

where $z$ is a coordinate normal to the bubble wall in the wall's rest frame, with $z>0(z<0)$ being the broken (symmetric) phase, and $v_{w}$ is the wall's velocity. $\bar{D}=\frac{1}{16}\left(9 D_{q}+7 D_{h}\right)$ in Eq. (16) is the effective diffusion constant defined in terms of quark $D_{q}$ and Higgs $D_{h}$ difussion constants. The strong sphaleron rate is given by $^{15} \Gamma_{s s}=4.9 \times 10^{-4} T$, while $k_{ \pm}=v_{w}\left(1 \mp \sqrt{1+4 \bar{D} \Gamma_{ \pm} / v_{w}^{2}}\right) / 2 \bar{D}$ where $\Gamma_{ \pm}$are the effective Higgs decay rate when $\rho$ assumes the respective values $v_{T_{c}}^{b r}$ or $v_{T_{c}}^{s}$. The $\mathcal{C P}$-violating ${ }^{16}$ source related to the Higgs-top anomalous interactions (4):

$$
S_{t}^{C P V}(z) \approx \frac{3}{2 \pi^{2}} T \gamma_{w} v_{w} m_{t}^{2}(z) \partial_{z} \theta_{t}(z)
$$


where $m_{t}(z)$ and $\theta(z)$ are the modulus and phase of the complex top quark mass:

$$
\begin{aligned}
m_{t}(z) & =\sqrt{\left(m_{t}^{\prime}+\frac{y_{t}}{\sqrt{2}} \rho(z) \cos \xi\right)^{2}+\left(\frac{y_{t}}{\sqrt{2}} \rho(z) \sin \xi\right)^{2}} \\
\tan \theta_{t}(z) & =\frac{y_{t} \rho(z) \sin \xi}{\sqrt{2} m_{t}^{\prime}+y_{t} \rho(z) \cos \xi} \\
\rho(z) & =v_{T_{c}}^{b r}+\frac{v_{T_{c}}^{s}-v_{T_{c}}^{b r}}{2}\left[1+\tanh \left(\frac{z}{L_{w}}\right)\right] .
\end{aligned}
$$

where the bubble wall configuration $\rho(z)$ extrapolates between the broken vacuum $v_{T_{c}}^{b r}$ to the symmetric vacuum $v_{T_{c}}^{s}$. We note that the $\mathcal{C P}$-violation is defined by the phase $\xi$ in (4), $S_{t}^{C P V} \rightarrow 0$ as $\xi \rightarrow 0$. The wall width $L_{w}$ and the wall velocity, $v_{w}$ is not precisely determined by numerical calculation, but is instead kept as free parameters varying in the expected ranges $L_{w} \in\left[3 / T_{c}, 16 / T_{c}\right]$ and $v_{w} \in\left[10^{-3}, 1 / \sqrt{3}\right]$. The asymmetry parameter is then given by $\eta_{B}=n_{B} / s$, where $n_{B}$ is computed in Eq. (16) and $s=2 \pi^{2} g_{*} T^{3} / 45$ is the entropy density with $g_{*} \sim 100$ counting the effective number of relativistic degrees of freedom in equilibrium at temperature

Table 2. $\quad \eta_{B}$ computed with fixed $v_{w}=0.01$ and $L_{w}=3 / T_{c}$ and for $\xi=0.25 \pi$, various values of $y_{t}$ subject to constraints obtained in and $\kappa|v| / m_{h}^{2}=-0.5,-2$ (with $v=+246 \mathrm{GeV}$ and $m_{t}^{(+)}$). One can generate similiar $\eta_{B}$ by simultaneously reversing the signs of $\kappa$ and $v_{T_{c}}^{b r, s}$ and making the replacement $m_{t}^{\prime(+)} \rightarrow-m_{t}^{\prime(-)}$

\begin{tabular}{c|c|c|c|c}
\hline \multirow{2}{*}{} & \multirow{2}{*}{$\kappa|v| / m_{h}^{2}$} & \multicolumn{3}{|c}{$\xi=0.25 \pi$} \\
\cline { 3 - 5 } & & $y_{t}=0.62 y_{t}^{S M}$ & $y_{t}=0.69 y_{t}^{S M}$ & $y_{t}=0.76 y_{t}^{S M}$ \\
\hline \hline \multirow{2}{*}{$\eta_{B}=n_{B} / s$} & -0.5 & $9.28 \times 10^{-10}$ & $3.41 \times 10^{-9}$ & $2.41 \times 10^{-8}$ \\
\cline { 2 - 5 } & -2.0 & $2.02 \times 10^{-5}$ & $1.92 \times 10^{-5}$ & $1.38 \times 10^{-5}$ \\
\hline
\end{tabular}

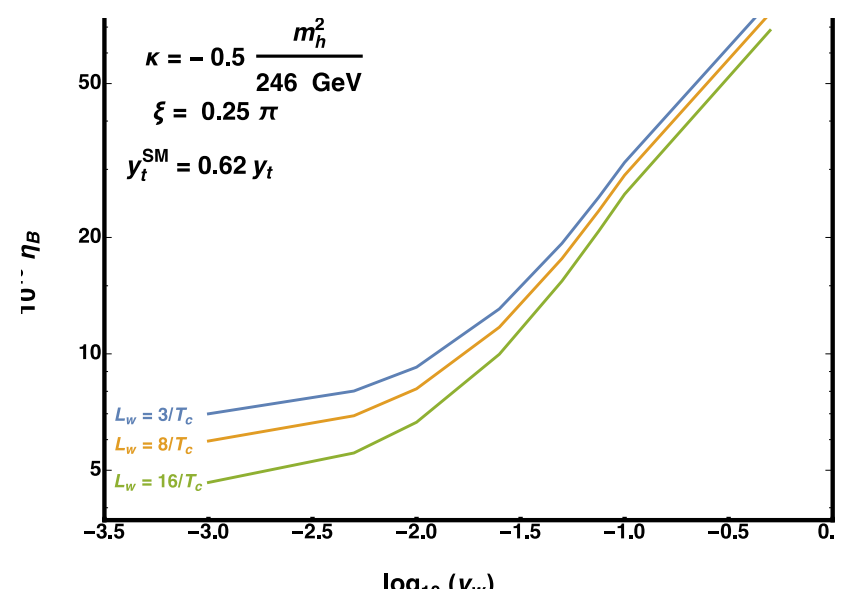

Fig. 2. The dependence of the baryon asymmetry parameter $\eta_{B}$ on the width of the bubble wall $L_{w}$ and its velocity $v_{w}$. The wall velocity is bounded $0.001 \lesssim v_{w} \lesssim 1 / \sqrt{3}$. 
$T$. We observe that a significant asymmetry can be produced assuming the Higgs anomalous couplings $\kappa, y_{t}$ and phase $\xi$. Tab. 2 and Fig. 2 shows that the asymmetry increases with the increase of the anomalous couplings $\kappa$ and $y_{t}$.

\section{Conclusion and outlook}

We studied the phase transition associated with the breaking of electroweak symmetry being non-linearly realised and and computed the baryon asymmetry. We have found the strong first-order phase transition is realised for a wide range of the anomalous couplings (see Tab. 1). The notable point of our scenario is that with the phase $\xi$ controlling the amount of $\mathcal{C} \mathcal{P}$-violation, there exist a finite range (15) of non-zero Higgs expectation value $v_{T_{c}}^{s}$ in the symmetric phase for which the sphaleron transitions are unsuppressed. The computed the baryon asymmetry parameter $\eta_{B}$ and can be comfortably accommodate the observed value (1) within the allowed range of parameters of the anomalous interactions (see Tab. 2 and Fig. 2). We ${ }^{6,17}$ and other authors ${ }^{18}$ have discussed measurement of the Higgs-top Yukawa in the $t \bar{t} h$ and $t h j$ channel but a sufficiently high luminosity will be needed to obtain sizeable sensitivity to the anomalous top-Yukawa couplings due to difficult QCD backgrounds. With the anomalous couplings favourable by our baryogenesis scenario, the trilinear couplings deviations results in deviations of the size $\delta_{\lambda_{3}}=\left[\frac{1}{6}, \frac{2}{3}\right] \lambda_{3}^{S M}$ from that of the SM, $\lambda_{3}^{S M}=\frac{m_{h}^{2}}{2 v}$. This lies in the expected precision of $25-50 \%$ that is anticipated at $14 \mathrm{TeV}$ high-luminosity $\left(3000 \mathrm{fb}^{-1}\right)$ LHC. ${ }^{19}$

\section{References}

1. G. Aad et al., Tech. Rep. ATLAS-CONF-2015-044, CERN (Geneva, 2015).

2. D. E. Morrissey and M. J. Ramsey-Musolf, New J. Phys. 14, p. 125003 (2012).

3. R. Cooke et al., Astrophys. J. 781, p. 31 (2014).

4. P. A. R. Ade et al., 1502.01589.

5. A. Kobakhidze, 1208.5180.

6. A. Kobakhidze, L. Wu and J. Yue, JHEP 10, p. 100 (2014).

7. A. Kobakhidze, L. Wu and J. Yue, 1512.08922.

8. J. Baron et al., Science 343, 269 (2014).

9. N. S. Manton, Phys. Rev. D28, p. 2019 (1983).

10. P. B. Arnold and L. D. McLerran, Phys. Rev. D36, p. 581 (1987).

11. L. Carson, X. Li, L. D. McLerran and R.-T. Wang, Phys. Rev. D42, 2127 (1990).

12. A. E. Nelson, D. B. Kaplan and A. G. Cohen, Nucl. Phys. B373, 453 (1992).

13. F. R. Klinkhamer and N. S. Manton, Phys. Rev. D30, p. 2212 (1984).

14. P. Huet and A. E. Nelson, Phys. Rev. D53, 4578 (1996).

15. G. D. Moore, Phys. Lett. B412, 359 (1997).

16. P. Huet and A. E. Nelson, Phys. Lett. B355, 229 (1995).

17. J. Yue, Phys. Lett. B744, 131 (2015).

18. J. Ellis, D. S. Hwang, K. Sakurai and M. Takeuchi, JHEP 04, p. 004 (2014).

19. C.-T. Lu, J. Chang, K. Cheung and J. S. Lee, JHEP 08, p. 133 (2015). 